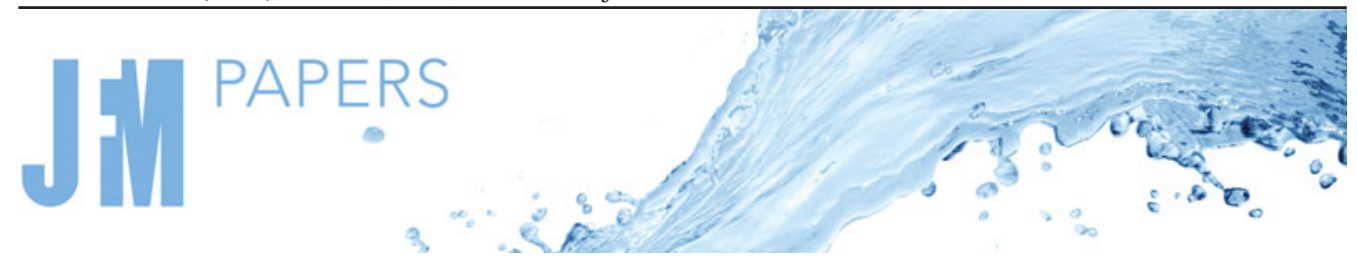

\title{
Much faster heat/mass than momentum transport in rotating Couette flows
}

\author{
Geert Brethouwer $\dagger$ \\ FLOW, Department of Engineering Mechanics, KTH, SE-100 44 Stockholm, Sweden
}

(Received 22 August 2020; revised 20 December 2020; accepted 23 December 2020)

Heat and mass transport are generally closely correlated to momentum transport in shear flows. This so-called Reynolds analogy between advective heat or mass transport and momentum transport hinders efficiency improvements in engineering heat and mass transfer applications. I show through direct numerical simulations that in plane Couette and Taylor-Couette flow, rotation can strongly influence wall-to-wall passive tracer transport and make it much faster than momentum transport, clearly in violation of the Reynolds analogy. This difference between passive tracer transport, representative of heat/mass transport, and momentum transport is observed in steady flows with large counter-rotating vortices at low Reynolds numbers as well as in fully turbulent flows at higher Reynolds numbers. It is especially large near the neutral (Rayleigh's) stability limit. The rotation-induced Coriolis force strongly damps the streamwise/azimuthal velocity fluctuations when this limit is approached, while tracer fluctuations are much less affected. Accordingly, momentum transport is much more reduced than tracer transport, showing that the Coriolis force breaks the Reynolds analogy. At higher Reynolds numbers, this strong advective transport dissimilarity is accompanied by approximate limit cycle dynamics with intense low-frequency bursts of turbulence when approaching the neutral stability limit. The study demonstrates that simple body forces can cause clear dissimilarities between heat/mass and momentum transport in shear flows.

Key words: rotating turbulence, Taylor-Couette flow

\section{Introduction}

Advective transport of heat and mass by fluid motions is fundamental to planetary and astrophysical processes and many engineering applications (Balbus \& Hawley 1998; Kays, Crawford \& Weigand 2005). Efficient advective transport contributes to energy savings in

$†$ Email address for correspondence: geert@mech.kth.se

(C) The Author(s), 2021. Published by Cambridge University Press. This is an Open Access article, distributed under the terms of the Creative Commons Attribution licence (http://creativecommons.org/ licenses/by/4.0/), which permits unrestricted re-use, distribution, and reproduction in any medium, provided the original work is properly cited. 


\section{G. Brethouwer}

buildings (Lake, Rezaie \& Beyerlein 2017), process industry (Keil 2018) and data centres (Alben 2017), and can be obtained by, e.g., applying wall roughness (Zhu et al. 2019) and flow control (Yamamoto, Hasegawa \& Kasagi 2013; Kaithakkal, Kametani \& Hasegawa 2020). In particular, optimal transport given minimal power input generates energy savings in applications (Alben 2017; Motoki, Kawahara \& Shimizu 2018), but optimization is challenging since flow vortices and eddies generally transport momentum and heat/mass at similar rates. This so-called Reynolds analogy between transport of momentum and heat/mass was postulated by Reynolds (1874), and applies to many shear flows (Kays 1994; Kays et al. 2005; Pirozzoli, Bernardini \& Orlandi 2016) including astrophysical flows (Guan \& Gammie 2009) when Prandtl numbers are close to unity (Ziefuß \& Mehdizadeh 2020). The Reynolds analogy is used for modelling advective transport in engineering (Kays et al. 2005), geophysical (Bretherton \& Park 2009) and astrophysical flows (Birnsteil, Dullemond \& Brauer 2010), but implies that higher heat/mass transfer goes together with higher momentum transfer and thus power input (Yamamoto et al. 2013).

In recent theoretical studies, incompressible steady flows are computed that maximize heat transfer for a given power input (Hassanzadeh, Chini \& Doering 2014; Alben 2017; Motoki et al. 2018; Souza, Tobasco \& Doering 2020). Motoki et al. (2018) consider plane Couette flow and show that the optimized flow has a much higher heat transfer for a given power input than ordinary turbulent flow. The computed optimized flows are not required to obey known momentum equations in these theoretical studies - that is, these optimal flows can be obtained applying a body force, but the body force can be arbitrary and does not (necessarily) have a familiar form. It is therefore not clear if this optimal transport is realizable, although Alben (2017) and Motoki et al. (2018) suggest that optimal flows can be approached by applying smart forcing or control techniques. I show through direct numerical simulations (DNSs) that in existing flows, namely incompressible plane Couette flow (PCF) and Taylor-Couette flow (TCF) subject to a Coriolis force, passive tracer transport can be much faster than momentum transport, in violation of the Reynolds analogy. It is thus possible to significantly change the ratio of wall-to-wall heat/mass to momentum transport by a simple Coriolis body force. Momentum transport in Couette flows has been explored extensively (Salewski \& Eckhardt 2015; Grossmann, Lohse \& Sun 2016) owing to its relevance for, e.g., astrophysics. TCF with heat or mass transport finds applications in, for example, cooling of electrical motors (Fénot et al. 2011) and chemical reactors and bioreactors (Nemri, Charton \& Climent 2016).

\section{Governing equations and numerical procedure}

TCF is a shear flow created between two rotating concentric cylinders, while PCF is the small-gap limit $d / r_{i} \rightarrow 0\left(\eta=r_{i} / r_{o} \rightarrow 1\right)$ of TCF, where $d$ is the gap between the cylinders/walls and $r_{i}$ and $r_{o}$ the inner and outer radii, respectively. In these flows I study passive tracer transport, mimicking heat and mass transport when the temperature/mass does not affect the flow. Hereafter, the passive tracer is called temperature for convenience, but the only body force affecting the flow is the Coriolis force, which does not perform any work.

Fluid motion and passive tracer transport in the PCF and TCF DNSs are governed by the Navier-Stokes and advection-diffusion equations,

$$
\frac{\partial \boldsymbol{U}}{\partial t}+\boldsymbol{U} \cdot \boldsymbol{\nabla} \boldsymbol{U}=-\nabla P+\frac{1}{R e} \nabla^{2} \boldsymbol{U}-R_{\Omega}\left(\boldsymbol{e}_{z} \times \boldsymbol{U}\right),
$$


Much faster heat/mass than momentum transport

$$
\frac{\partial T}{\partial t}+U \cdot \nabla T=\frac{1}{P r R e} \nabla^{2} T,
$$

together with $\nabla \cdot U=0$. The imposed azimuthal (streamwise) velocity and temperature at the inner and outer no-slip and iso-thermal walls, $\pm U_{w}$ and $\pm T_{w}$, respectively, are constant. Velocity $U$ is normalized by $U_{w}$, temperature $T$ by $T_{w}$ and length by $d$. The modified non-dimensional pressure $P$ includes the centrifugal force (Salewski \& Eckhardt 2015). The rotation axis, defined by the unit vector $\boldsymbol{e}_{z}$, is the spanwise and central axis in PCF and TCF, respectively, as in Brauckmann, Salewski \& Eckhardt (2016), and is parallel with the mean flow vorticity. Sketches of the flow geometries are presented in the supplementary material available at https://doi.org/10.1017/jfm.2020.1176. A Reynolds number $R e=\Delta U d / v$ and rotation number $R_{\Omega}=2 \Omega d / \Delta U$, where $\Delta U=2 U_{w}, v$ is the kinematic viscosity and $\Omega$ is the imposed system rotation, characterize the flow. $R_{\Omega}$ is defined such that it is negative for cyclonic flows (same sign for shear and rotation) and positive for anti-cyclonic flows. These parameters are equivalent to the shear Reynolds and rotation numbers used by Dubrulle et al. (2005) and Brauckmann et al. (2016). The rotating reference frame for TCF can naturally be translated back to a laboratory reference frame (Ezeta et al. 2020). $\operatorname{Pr}=v / \alpha$ is the Prandtl number with $\alpha$ the thermal diffusivity.

From (2.1) and (2.2), it follows that in PCF the wall-to-wall mean dimensionless momentum $J^{m}=\langle U V\rangle-\partial_{y}\langle U\rangle / \operatorname{Re}$ and heat fluxes $J^{h}=\langle V T\rangle-\partial_{y}\langle T\rangle /(\operatorname{RePr})$ are conserved, and in TCF the angular velocity flux $J^{m}=r^{3}\left(\langle V \omega\rangle-\partial_{r}\langle\omega\rangle / R e\right)$ and heat current $J^{h}=r\left[\langle V T\rangle-\partial_{r}\langle T\rangle /(\operatorname{RePr})\right]$ are conserved (Brauckmann et al. 2016). Here, $\omega=U / r$ is the angular velocity, $U$ and $V$ the streamwise (azimuthal) and wall-normal (radial) velocity, and $\langle\cdots\rangle$ denotes averaging over time and area at constant wall-normal (radial) distance in PCF (TCF). The Nusselt numbers $N u_{m}=J^{m} / J_{\text {lam }}^{m}$ and $N u_{h}=J^{h} / J_{\text {lam }}^{h}$ quantify the wall-to-wall (angular) momentum transport (Brauckmann et al. 2016) and heat transport, respectively. The subscript 'lam' implies the molecular (conductive) flux for laminar flow. For laminar PCF, $J_{\text {lam }}^{m}=-1 / \operatorname{Re}$ and $J_{\text {lam }}^{h}=-1 / \operatorname{RePr}$. For laminar $\mathrm{TCF}, J_{\text {lam }}^{m}=-2 \eta /\left(\operatorname{Re}(1-\eta)^{2}\right)\left(\right.$ Brauckmann et al. 2016) and $J_{\text {lam }}^{h}=2 /(\operatorname{RePr} \ln \eta) . N u_{m}$ specifies the force (torque) needed to shear the flow in units of that in laminar PCF (TCF), and $N u_{h}$ is the heat flux in units of that in laminar flow.

To study the influence of Coriolis forces on momentum and heat transfer, I have carried out several DNS series at constant $R e$ up to 40000 with varying $R_{\Omega}-$ i.e. eight DNS series of PCF at a constant $R e=240,400,800,1600,3200,6400,17200$ and 40000, respectively, and eight DNS series of TCF at a constant $R e=400,1152,2593,3889,8750$, 19688, 29531 and 40000 , respectively, all at varying $R_{\Omega}$. The supplementary material presents tables with $R e$ and $R_{\Omega}$ parameters of all DNSs. In PCF, $P r=1$ and in TCF, $\operatorname{Pr}=0.7$ and $\eta=0.714$.

The governing equations for PCF are solved with a Fourier-Fourier-Chebyshev algorithm, with periodic boundary conditions in the streamwise and spanwise directions (Chevalier et al. 2014). The computational domain sizes are $6 \pi d$ and $2 \pi d$ in the streamwise and spanwise directions, respectively, which is large enough to accommodate several pairs of counter-rotating large-scale vortices. The governing equations for TCF in cylindrical coordinates are solved with a Fourier-Fourier-finite-difference algorithm (Boersma 2011; Peeters et al. 2016), with periodic boundary conditions in the axial and azimuthal directions. In the radial direction, a sixth-order compact-finite-difference scheme is used. Like others, I do not simulate the flow around the entire cylinder but use a domain with reduced size in the azimuthal direction. Previously, it has been verified that changing the domain size has little effect on the computed torque (Brauckmann \& Eckhardt 2013; 


\section{G. Brethouwer}

$\begin{array}{lcccccccc}R e & 400 & 1152 & 2593 & 3889 & 8750 & 19688 & 29531 & 40000 \\ L_{\theta} / d & 6 \pi & 6 \pi & 6 \pi & 6 \pi & \pi & \pi & \pi & 3 \pi \\ L_{z} / d & 2 & 2 & 2 & 2 & 2 & 2 & 2 & 4 \pi / 3\end{array}$

Table 1. Domain size in the DNSs of TCF. $L_{\theta}$ is the azimuthal domain size at the centreline, and $L_{z}$ is the axial domain size.

Brauckmann et al. 2016). The computational domain size in the DNSs of TCF, listed in table 1, is basically the same as in the DNSs of Brauckmann \& Eckhardt (2013) up to $R e=29531$ and wide enough to capture at least one pair of counter-rotating Taylor vortices. In the DNSs at $R e=40000$, the domain is significantly larger.

The resolution increases with $R e$ to keep the grid spacing in terms of viscous wall units within acceptable bounds. At $R e=40000$, the streamwise and spanwise grid spacings in the PCF DNSs are $\Delta x^{+} \leq 13$ and $\Delta z^{+} \leq 6.5$, respectively, and in the TCF DNSs, the azimuthal and axial grid spacings are $\Delta x^{+}=12.4$ and $\Delta z^{+}=5.9$, respectively at $R_{\Omega}=$ 0.3 and smaller at higher $R_{\Omega}$. This is the grid spacing in terms of Fourier modes and viscous wall units, comparable to the grid spacing in other well-resolved DNSs of wall flows (Lee \& Moser 2015). The number of Chebyshev modes or radial grid points with near-wall clustering is 192 or more at this $R e$.

The DNSs of PCF and TCF are either initialized with perturbations to trigger vortices or with the fields of a DNS at another $R_{\Omega}$. They are run for a sufficiently long time to reach a statistically stationary state and then run for a long period to obtain well-converged statistics. I have verified that the Nusselt numbers do not change when the DNSs are continued. For several DNSs of PCF, I have also validated that changing the domain size and resolution does not affect the results. The DNS results of TCF for $N u_{m}$ agree well with previous DNSs of Brauckmann \& Eckhardt (2013) and Ostilla et al. (2013). These validations are presented in the supplementary material.

\section{Results}

Figure $1(a, b)$ shows that momentum transfer in terms of $N u_{m}$ naturally grows with $R e$ but also varies with $R_{\Omega}$ in the DNSs owing to changing flow features (Salewski \& Eckhardt 2015; Grossmann et al. 2016). At $R_{\Omega}=0$, PCF is linearly stable yet turbulent when $R e \gtrsim 1600$ owing to subcritical transition and therefore $N u_{m}>1$. TCF is linearly unstable and $N u_{m}>1$ if $R_{\Omega}=0$ at all $R e$ (Dubrulle et al. 2005). In both flows, $N u_{m}$ first grows with $R_{\Omega}$ due to destabilization by anticyclonic rotation and then declines towards unity for $R_{\Omega} \rightarrow 1$ when the flow approaches the linearly stability limit $R_{\Omega}^{c}$ and relaminarizes (Dubrulle et al. 2005). Disturbances and turbulence cannot sustain beyond $R_{\Omega}^{c}$, even at higher $\operatorname{Re}$ (Ostilla-Mónoci et al. 2014b). Momentum transport is maximal around $R_{\Omega}=0.2$ in PCF and around $R_{\Omega}=0.3$ to 0.1 at low to high $R e$ in TCF, consistent with previous numerical (Brauckmann \& Eckhardt 2013; Salewski \& Eckhardt 2015; Brauckmann et al. 2016) and experimental observations (van Gils et al. 2011). This broad maximum is linked to intermittent bursts in the outer layer in TCF and to strong vortical motions in PCF (Brauckmann \& Eckhardt 2013; Brauckmann et al. 2016). Another narrow maximum in $N u_{m}$ caused by shear instabilities appears in PCF at $R_{\Omega} \approx 0.02$ when $R e \gtrsim 10^{4}$ (Brauckmann \& Eckhardt 2013), but my DNSs do not cover this narrow region near $R_{\Omega}=0$ and therefore do not reveal this second maximum. With rising $R e$, the narrow 

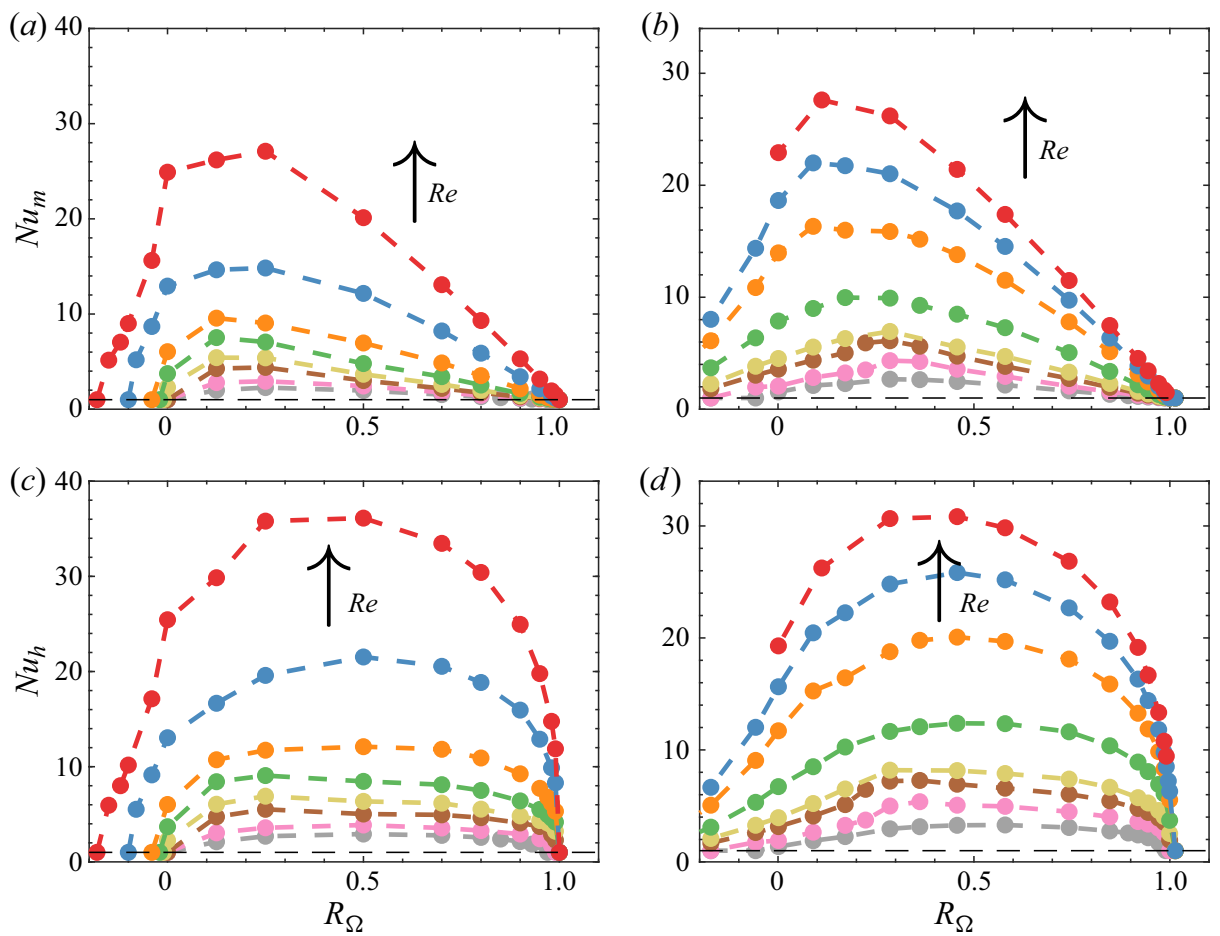

Figure 1. (a) $N u_{m}$ and (c) $N u_{h}$ for PCF. (b) $N u_{m}$ and (d) $N u_{h}$ for TCF. Each line/colour represents a different constant $R e$, and dots denote DNS results. The horizontal dashed line marks $N u_{m}, N u_{h}=1$. Arrows show trends for increasing $R e$ listed in $\S 2$.

maximum overtakes the broad maximum, which disappears in TCF with $\eta=0.91$ if $R e$ is higher than in my DNSs (Ezeta et al. 2020).

Heat transfer in terms of $N u_{h}$ behaves similarly to $N u_{m}$ at low $R_{\Omega}$ but differently at higher $R_{\Omega}$ (figure $1 c, d$ ). Its maximum is higher and at higher $R_{\Omega}$ for almost all $R e$, demonstrating that flow structures causing optimal momentum transport do not necessarily cause optimal heat transport. At higher $R e, N u_{h}$ is maximal near $R_{\Omega}=0.5$ in both PCF and TCF and then sharply declines when $R_{\Omega} \rightarrow 1$ and the flow relaminarizes. This means that in a laboratory instead of a rotating frame of reference, maximal momentum transfer in higher $R e$ TCF happens with moderate counter-rotation, whereas maximal heat transfer happens with co-rotating inner and outer cylinders. The growths of the maximum $N u_{m}$ and $N u_{h}$ over all $R_{\Omega}$ at fixed $R e$ show similar trends with $R e$ in PCF and TCF and follow $N u_{m}, N u_{h} \sim$ $R e^{0.6}$ at higher $\operatorname{Re}$ (figure 2). Experiments show that for $\operatorname{Re}>3 \times 10^{4}$, the scaling $N u_{m} \sim$ $R e^{0.78}$ for all $R_{\Omega}$ in TCF (Ostilla-Mónoci et al. 2014a,b). It is therefore possible that at higher $R e$, the maximum $N u_{h}$ follows a different scaling than $N u_{h} \sim R e^{0.6}$ observed here.

The ratio HTE $=N u_{h} / N u_{m}$, shown in figure 3, is a measure of heat transfer efficiency since $N u_{m}$ is proportional to the power input (van Gils et al. 2011).An equivalent measure is considered in heat transfer optimization studies by Yamamoto et al. (2013), Motoki et al. (2018) and Kaithakkal et al. (2020). A high similarity between momentum and heat transport can be expected at $R_{\Omega}=0$ in PCF because $P r=1$, and momentum and heat transport are similarly forced. This is vindicated by the DNSs: the difference between $N u_{h}$ and $N u_{m}$ is not more than $2 \%$ at all $R e$ and accordingly HTE $\simeq 1$, meaning that the Reynolds analogy perfectly applies. HTE is somewhat smaller in TCF at $R_{\Omega}=0$ because 


\section{G. Brethouwer}

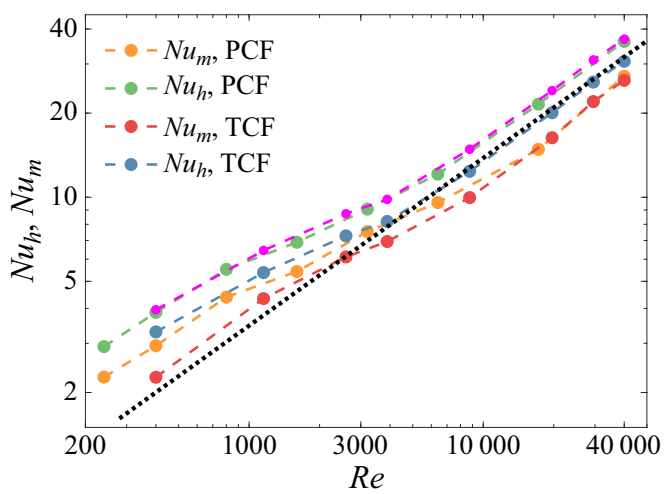

Figure 2. Maximum value of $N u_{m}$ and $N u_{h}$ over $R_{\Omega}$ at fixed $R e$ in PCF and TCF as function of $R e$. The dotted black line shows the scaling $N u \sim R e^{0.6}$.

$\operatorname{Pr}<1$, but it is still near unity so that the Reynolds analogy approximately holds. Clear differences in heat and momentum transport emerge for increasing $R_{\Omega}$. HTE rapidly grows with $R_{\Omega}$ in PCF and TCF and reaches a maximum around $R_{\Omega} \approx 0.85-0.99$ at low to high $R e$ before abruptly dropping to unity for $R_{\Omega} \geq 1$. Its maximum grows from about two at the lowest $R e$ to eight and more than six at $R e=40000$ in PCF and TCF, respectively (figures 3 and $4 a$ ). In TCF, the maximum HTE seems to level off at higher Re, while in PCF it continues to grow. TCF with a fixed outer and rotating inner cylinder in a laboratory frame corresponds to $R_{\Omega}=1-\eta=0.29$ and has a HTE $\leq 1.18$ over all $R e$ considered here, much less than the maximum possible HTE. Note that Couette flow is linearly unstable very near $R_{\Omega}=1$ (Nagata 1990; Esser \& Grossmann 1996), so flow motions can be sustained even very near $R_{\Omega}=1$. Figure $4(b)$ shows that the HTE has a similar trend in PCF and TCF near $R_{\Omega}=1$ for $R e \geq 17200$. DNS results for TCF at the three $R e$ are hardly distinguishable since they overlap, although the computational domain sizes are different, suggesting the results are indifferent to this aspect. Some of the dissimilarities between heat transfer in PCF and TCF can be attributed to the different values of $\mathrm{Pr}$ in the two cases. DNSs of heat transfer in turbulent channel flow support that $\mathrm{Nu}_{h} \sim \mathrm{Pr}^{1 / 2}$ for $\mathrm{Pr}$ near unity (Pirozzoli et al. 2016), indicating that $N u_{h}$ and HTE are a factor $0.7^{-1 / 2}$ larger in TCF if $P r=1$, as in PCF, instead of 0.7 , as used here. The differences observed in, e.g., figures 2 and 4 between PCF and TCF results indeed largely disappear (not shown here), and $N u_{h}$ is almost unity in TCF at $R_{\Omega}=0$ after this scaling.

Results for HTE can be compared to theoretical optimal heat transport for $\operatorname{Pr}=1$ in PCF with an arbitrary body force calculated by Motoki et al. (2018), who maximized heat and momentum transport dissimilarity by optimizing the ratio of total scalar dissipation to total energy dissipation $\varepsilon_{S} / \varepsilon_{E}$. For rotating PCF, with no energy input by the Coriolis force, HTE is equivalent to $\varepsilon_{S} / \varepsilon_{E}$. If $R e \leq 800$ and rotating PCF is streamwise-invariant as discussed later, $\varepsilon_{S} / \varepsilon_{E}$ calculated by Motoki et al. (2018) is nearly equal to the maximal HTE found here, implying that heat transport in rotating PCF is near optimal. At higher $R e$, results diverge: the optimal $\varepsilon_{S} / \varepsilon_{E}$ calculated by Motoki et al. (2018) at $R e=1600$, 6400 and 40000 , shown in their figure 7, is respectively 1.6, 2.6 and 3.1 times higher than the maximal HTE in rotating PCF. Their optimal heat transfer rate is also higher than in turbulent PCF without body forces. Thus, even though the dissimilarity between heat and momentum can be large in rotating PCF, it is theoretically possible to enhance it further through other body forces. Another factor which may contribute to the observed difference 

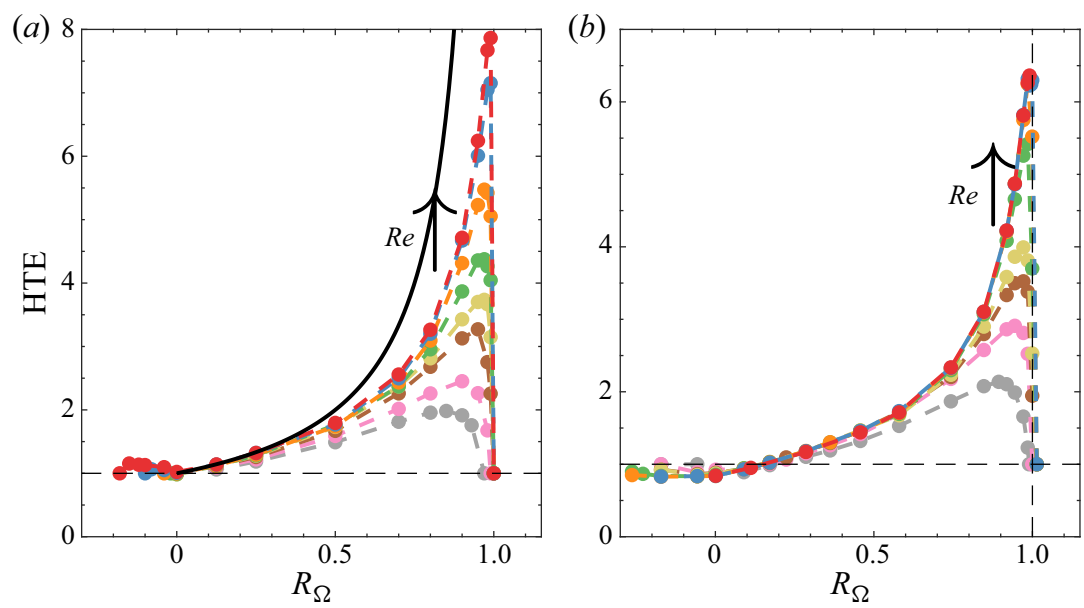

Figure 3. HTE as function of $R_{\Omega}$ in $(a) \mathrm{PCF}$ and $(b)$ TCF. Each line/colour represents a different constant $R e$. Arrows show trends for increasing $R e$ listed in $\S 2$, and dots signify DNS results. The black line in $(a)$ shows the high-Re limit.
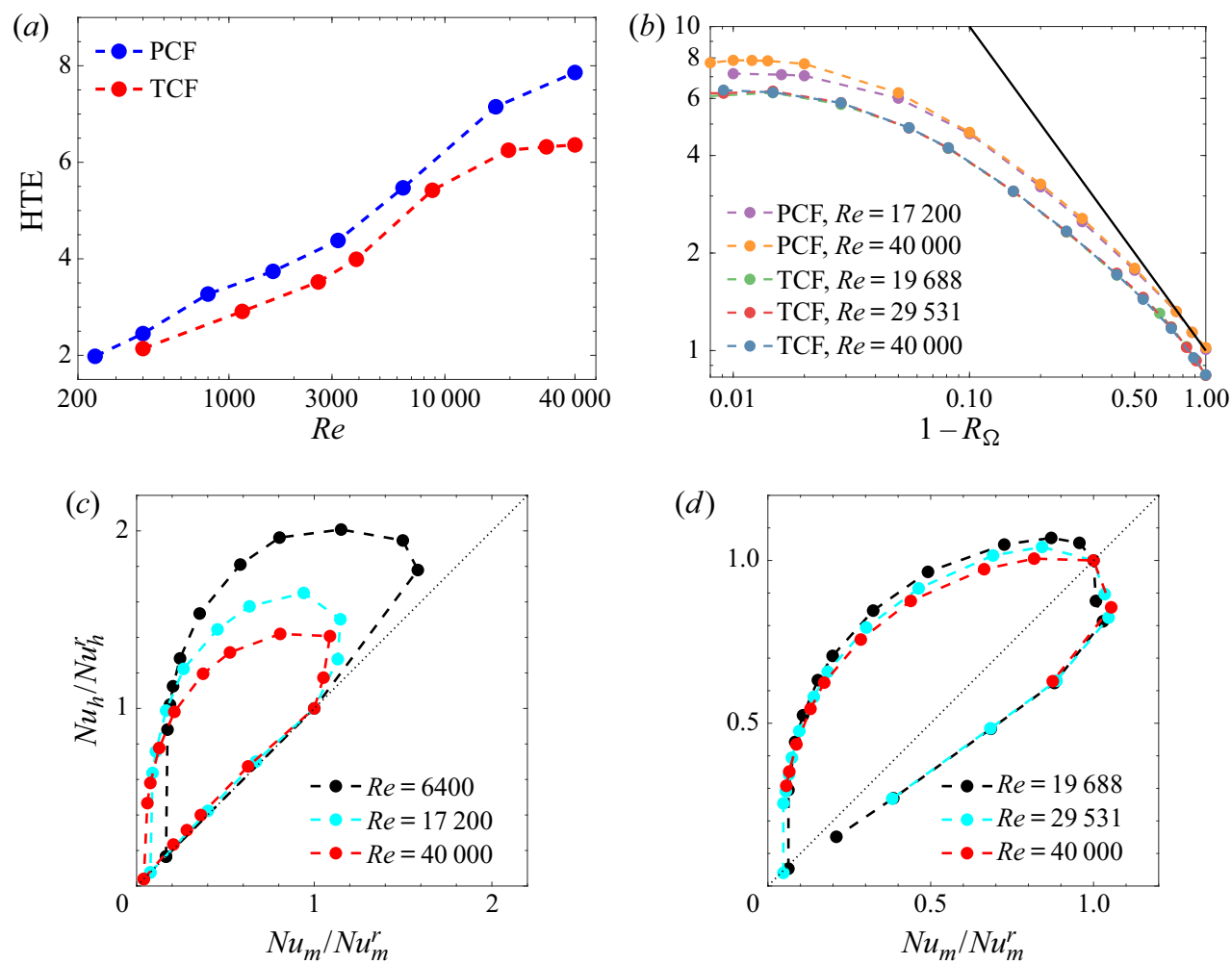

(d)

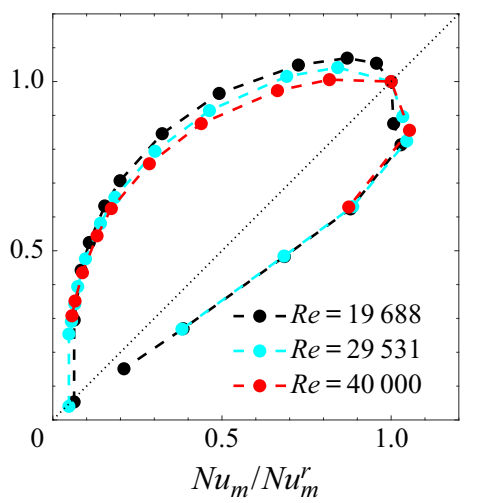

Figure 4. (a) Maximum HTE over $R_{\Omega}$ at fixed $R e$ in PCF and TCF as function of $R e$. (b) Log-log plot of HTE versus $1-R_{\Omega}$ in PCF at $R e=17200$ and 40000 and TCF at $R e=19688,25531$ and 40000 . The black solid line shows the high-Re limit HTE $=1 /\left(1-R_{\Omega}\right) . N u_{h} / N u_{h}^{r}$ versus $N u_{m} / N u_{m}^{r}$ at fixed $\operatorname{Re}$ for $(c)$ PCF and $(d)$ TCF. $N u_{m, h}^{r}$ is the reference Nusselt number corresponding to the non-rotating case $\left(R_{\Omega}=0\right)$ in PCF and the fixed outer cylinder case $\left(R_{\Omega}=1-\eta=0.29\right)$ in TCF. $R_{\Omega}$ increases in the counterclockwise direction. 


\section{G. Brethouwer}
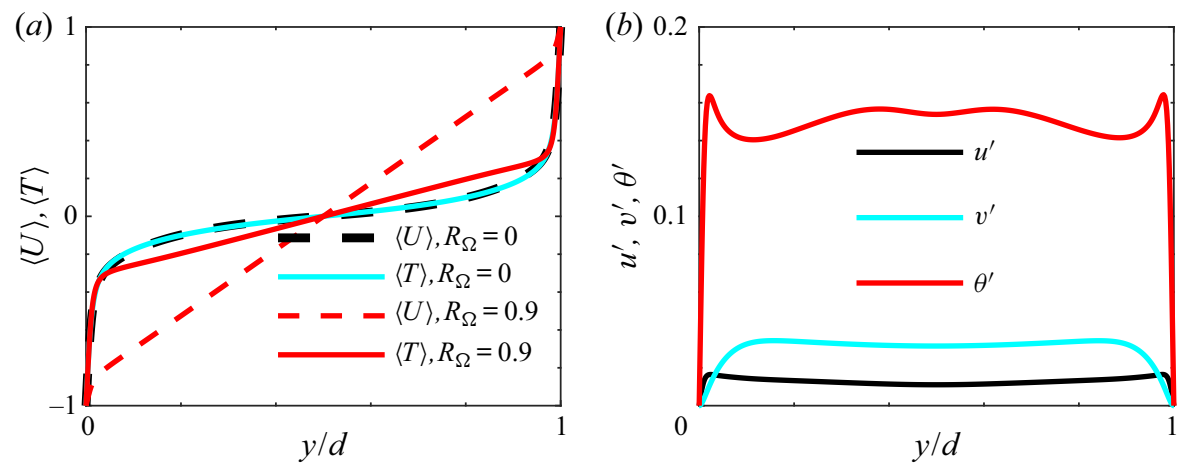

Figure 5. (a) Profiles of $\langle U\rangle$ and $\langle T\rangle$ in PCF at $R e=40000$ and $R_{\Omega}=0$ and 0.9. (b) Profiles of the root-mean-square of the streamwise and wall-normal velocity fluctuations $u^{\prime}$ and $v^{\prime}$, respectively, and temperature fluctuation $\theta^{\prime}$ in $\mathrm{PCF}$ at $\left(R e ; R_{\Omega}\right)=(40000 ; 0.98)$. Mean and fluctuating velocity and temperature are scaled by $U_{w}$ and $T_{w}$, respectively, and $y$ is the distance to the wall ( $y / d=0$ or 1 at the walls).

is that rotating PCF is unsteady and turbulent for $R e>800$, whereas Motoki et al. (2018) only considered optimal heat transport in steady PCF.

HTE defines the heat to momentum transfer ratio. In applications of advective heat $/$ mass transfer, one may seek to optimize other variables owing to constraints (Webb 1981; Hesselgreaves 2000; Yamamoto et al. 2013) - for example, to enhance heat transfer for equal power input or to reduce power input for equal heat transfer. Figure $4(c, d)$ therefore show $N u_{h} / N u_{h}^{r}$ versus $N u_{m} / N u_{m}^{r}$ for PCF and TCF at fixed $R e$ and varying $R_{\Omega}$ for the highest Re. Here, $N u_{m, h}^{r}$ are Nusselt numbers in the reference case at the same Re. For PCF the non-rotating case $\left(R_{\Omega}=0\right)$ is taken as reference, whereas for TCF the fixed outer cylinder case in a laboratory frame $\left(R_{\Omega}=1-\eta=0.29\right)$ is taken as reference because of its experimental relevance. Cases left of the dotted line have a higher HTE than the reference case. Some $R_{\Omega}$ cases in PCF have the same wall-to-wall momentum transfer $\left(N u_{m} / N u_{m}^{r} \simeq 1\right)$ but much higher heat transfer per unit area $\left(N u_{h} / N u_{h}^{r}>1\right)$, or the same heat transfer $\left(N u_{h} / N u_{h}^{r} \simeq 1\right)$ but much lower momentum transfer $\left(N u_{m} / N u_{m}^{r}<1\right)$ than at $R_{\Omega}=0$ (figure $4 c$ ). At $R e=40000$ and $R_{\Omega}=0.5, N u_{h} / N u_{h}^{r}=1.4$ and $N u_{m} / N u_{m}^{r}=0.8$, giving a $\mathrm{HTE}=1.8$, and at $R_{\Omega}=0.9, N u_{h} / N u_{h}^{r} \simeq 1$ and $N u_{m} / N u_{m}^{r} \simeq 0.21$, giving a $\mathrm{HTE}=4.7$. In TCF only a few cases have a somewhat higher heat transfer and/or lower momentum transfer than the reference case (figure $4 d$ ) since $N u_{h}$ is already high at $R_{\Omega}=0.29$ (figure $1 d$ ). However, HTE can be much higher than 1.18 as in the reference case, as shown before. When $\eta$ increases, we can expect $N u_{h} / N u_{h}^{r}$ versus $N u_{m} / N u_{m}^{r}$ curves for TCF to resemble curves for PCF if the fixed outer cylinder case is taken as reference since this case corresponds to $R_{\Omega}=1-\eta$, which approaches zero.

In anticyclonic rotating Couette flows, heat is thus transported much faster than momentum, in violation of the Reynolds analogy, when approaching the linear stability limit $R_{\Omega}^{c} \simeq 1$. Mean velocity and temperature profiles reflect the transport anomaly: at $R_{\Omega}=0$ these are barely distinguishable in $\mathrm{PCF}$, but when $R_{\Omega} \rightarrow 1$ the mean temperature $\langle T\rangle$ has a thin boundary layer and nearly linear centre profile and clearly differs from the mean streamwise velocity $\langle U\rangle$, which approaches the linear laminar profile (figure $5 a$ ). Here, $\langle\cdot\rangle$ denotes averaging over time and wall-parallel planes. Mean velocity and temperature profiles in TCF are not shown but behave similarly. The thermal boundary layer becomes thinner with $R e$ and changes more rapidly than the velocity boundary layer for $R_{\Omega}$ near $R_{\Omega}^{c}$, leading to a growth of the maximum HTE. 
Insight into the transport anomaly and small streamwise velocity fluctuations and related weak momentum transport at high $R_{\Omega}$ is obtained by studying the action of the Coriolis force. Consider the mean shear and Coriolis force term in the governing equation for $u$ in PCF, that is $v\left(2 \Omega-\partial_{y}\langle U\rangle\right)$, where $u$ and $v$ are the streamwise and wall-normal velocity fluctuations, respectively; if $\Omega>0$, the Coriolis force reduces production of $u$ by mean shear when $v \neq 0$. Note that the absolute mean vorticity $\partial_{y}\langle U\rangle-2 \Omega \approx 0$ about the channel centre at sufficiently high $R_{\Omega}$ (Brauckmann et al. 2016; Kawata \& Alfredsson 2016) and in the whole channel if $R_{\Omega} \rightarrow 1$. The Coriolis term in the Reynolds stress transport equation of $\langle u u\rangle$ then counterbalances the production term, and the only term producing $\langle u u\rangle$ is the pressure-strain correlation (Brethouwer 2017). If a fluid particle is displaced in the wall-normal direction by vortical motions, the Coriolis force basically accelerates or decelerates the particle so that its streamwise velocity approaches the local mean velocity. Figure 5(b) confirms that $u^{\prime}=\langle u u\rangle^{1 / 2}$ is small in PCF if $R_{\Omega} \rightarrow 1$, while $v^{\prime}=\langle v v\rangle^{1 / 2}$ is larger because vortical motions survive as long as $R_{\Omega}<R_{\Omega}^{c}$ and produce a high heat flux and intense temperature fluctuations that are not directly affected by the Coriolis force. Observations in TCF (not shown) are again similar: the specific angular momentum is nearly constant if $R_{\Omega} \rightarrow 1$, implying neutral stability according to Rayleigh's criterion (Brauckmann et al. 2016) and strongly reduced azimuthal velocity fluctuations.

Steady streamwise-invariant Taylor vortices are present at low $R e$. These are seen in visualizations of the flow field (not shown here) and indicated by the visualizations of the temperature field in figure 6(a,b). These vortices appear above the stability limit (Nagata 1990; Esser \& Grossmann 1996) and echo structures producing optimal heat transport in theoretical studies of PCF (Motoki et al. 2018). They transport considerable heat but little momentum since streamwise velocity fluctuations are small when $R_{\Omega} \rightarrow 1$, as discussed above. For streamwise-invariant PCF with $\operatorname{Pr}=1$, one can further quantify this and derive from (2.1) and (2.2)

$$
\hat{u} / U_{w}=\left(1-R_{\Omega}\right) \hat{\theta} / T_{w},
$$

where $\hat{u}$ and $\hat{\theta}$ are the streamwise velocity and temperature deviations from the laminar situation, respectively. Further, using a variable transformation as in Zhang et al. (2019) gives

$$
\mathrm{HTE}=1+\frac{R_{\Omega}}{1-R_{\Omega}} \frac{N u_{m}-1}{N u_{m}} .
$$

Eckhardt, Doering \& Whitehead (2020) derive exact relations between heat and momentum transport in two-dimensional Rayleigh-Bénard convection and rotating PCF that are equivalent to (3.2). Relations (3.1) and (3.2) are exact as long as $R e \leq 800$ and PCF is streamwise-invariant. Equation (3.1) shows that $\hat{u}$ declines relative to $\hat{\theta}$ when $R_{\Omega} \rightarrow 1$; and (3.2) shows that HTE $=1$ if $R_{\Omega}=0$, but HTE $>1$ if $0<R_{\Omega}<1$ and $N u_{m}>1$, so heat is transported more efficiently than momentum. Equation (3.2) further suggests a growing HTE with $R e$ as $N u_{m}$ increases. In the high $R e$-limit, $N u_{m} \rightarrow$ $\infty$, and consequently HTE $\rightarrow 1 /\left(1-R_{\Omega}\right)$ for streamwise-invariant PCF. The simulated HTE is lower because of finite $R e$ and turbulence, but for $0 \leq R_{\Omega} \leq 0.25$ when quasi two-dimensional streamwise vortices dominate transport (Brauckmann et al. 2016), this high-Re limit, shown by the black solid line in figure 3(a), closely matches DNSs.

In TCF both curvature and rotation play a role. In the rotating reference frame, the centripetal/Coriolis acceleration terms caused by streamline curvature in the non-dimensional equations of motion for TCF in cylindrical coordinates scale with the curvature number $R_{C}=(1-\eta) / \sqrt{\eta}-$ see the supplementary material and 


\section{G. Brethouwer}

(a)

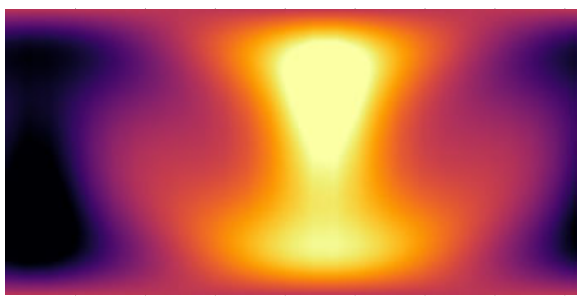

(c)

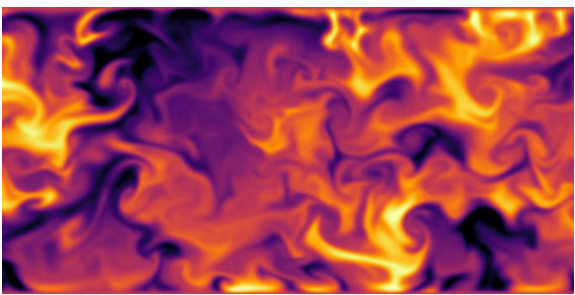

(b)

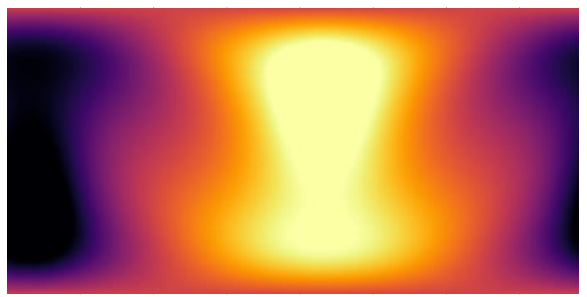

$(d)$

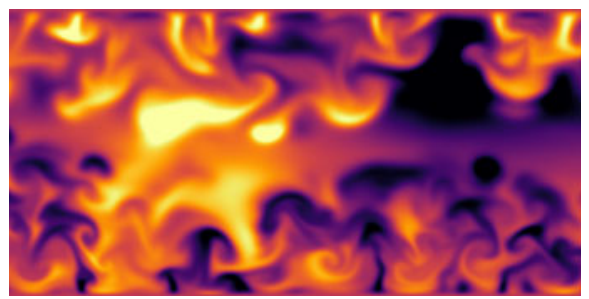

Figure 6. Snapshot of the instantaneous temperature fluctuation field at $(a, b) R e=400$ and $R_{\Omega}=0.9$ and $(c, d) R e=40000$ and $R_{\Omega}=0.99$ in $(a, c)$ PCF and $(b, d)$ TCF in a cross-stream plane. In $(b)$ the full and in $(a, c, d)$ a part of the spanwise/axial domain is shown.

Dubrulle et al. (2005) and Brauckmann et al. (2016). Curvature effects should therefore disappear if $\eta \rightarrow 1$ and consequently $R_{C} \rightarrow 0$. TCF properties are indeed similar to those of PCF, and profiles of $N u_{m}$ as a function of $R_{\Omega}$ collapse for $\eta \geq 0.9$ if $R e$ is equal, see Brauckmann et al. (2016), confirming that $R e$ and $R_{\Omega}$ appropriately describe Couette flows and the TCF to PCF transition. We can then also expect heat transfer in PCF and TCF with $\eta \geq 0.9$ to be similar if both $R e$ and $R_{\Omega}$ are equal. By contrast, when $\eta<0.9$ and $R_{\Omega}$ is low, curvature effects are important. TCF is then continuously turbulent in the inner partition while strongly intermittent in the outer partition due to a stabilizing influence of curvature (see Brauckmann et al. (2016)), and this affects momentum transfer.

However, beyond a critical $R_{\Omega}$, TCF is fully turbulent in the outer partition as well, and curvature effects are again less important. Consider the ratio of the curvature and rotation terms in the equations of motion given by $R_{C} U /\left(2 R_{\Omega}\right)$, where $U$ is the streamwise velocity scaled by $U_{w}$ - see the supplementary material for details. When $R_{\Omega}$ is sufficiently high, this ratio is less than unity, and therefore rotation influences should dominate even if $\eta<0$.9. For $R_{\Omega} \gtrsim 0.2, N u_{m}$ collapses for $\eta \geq 0.71$ and $R e$ as well as $R_{\Omega}$ are equal, as shown by Brauckmann et al. (2016) for moderate Re, which agrees with that idea. Also in the present study, $N u_{m}$ as function of $R_{\Omega}$ in PCF and TCF collapse for $R e=400$ and 40000 and $R_{\Omega} \gtrsim 0.3$. This is not explicitly shown but can be inferred by comparing figures $1(a)$ and $1(b)$. In the other cases $R e$ is different, which complicates a comparison. When rotation influences dominate and $N u_{m}$ is similar, we can expect that $N u_{h}$ is also similar in PCF and TCF. That appears to be true for the present cases once the differences in $\operatorname{Pr}$ have been accounted for. For lower $R_{\Omega}$, curvature effects are noticeable and cause differences in $N u_{m}$ and $N u_{h}$ in TCF and PCF, see figure 1. HTE seems to be less affected by curvature for $R_{\Omega} \geq 0$, although it could possibly cause a difference in heat and momentum transfer.

To summarize, streamline curvature has a noticeable effect on heat and mass transfer when it partly stabilizes TCF for $\eta<0.9$ and sufficiently low $R_{\Omega}$. When $\eta \geq 0.9$ or when $R_{\Omega}$ is sufficiently high and stabilization of TCF in the outer partition does not occur, curvature effects appear to have a small or negligible influence on heat and momentum transfer. 

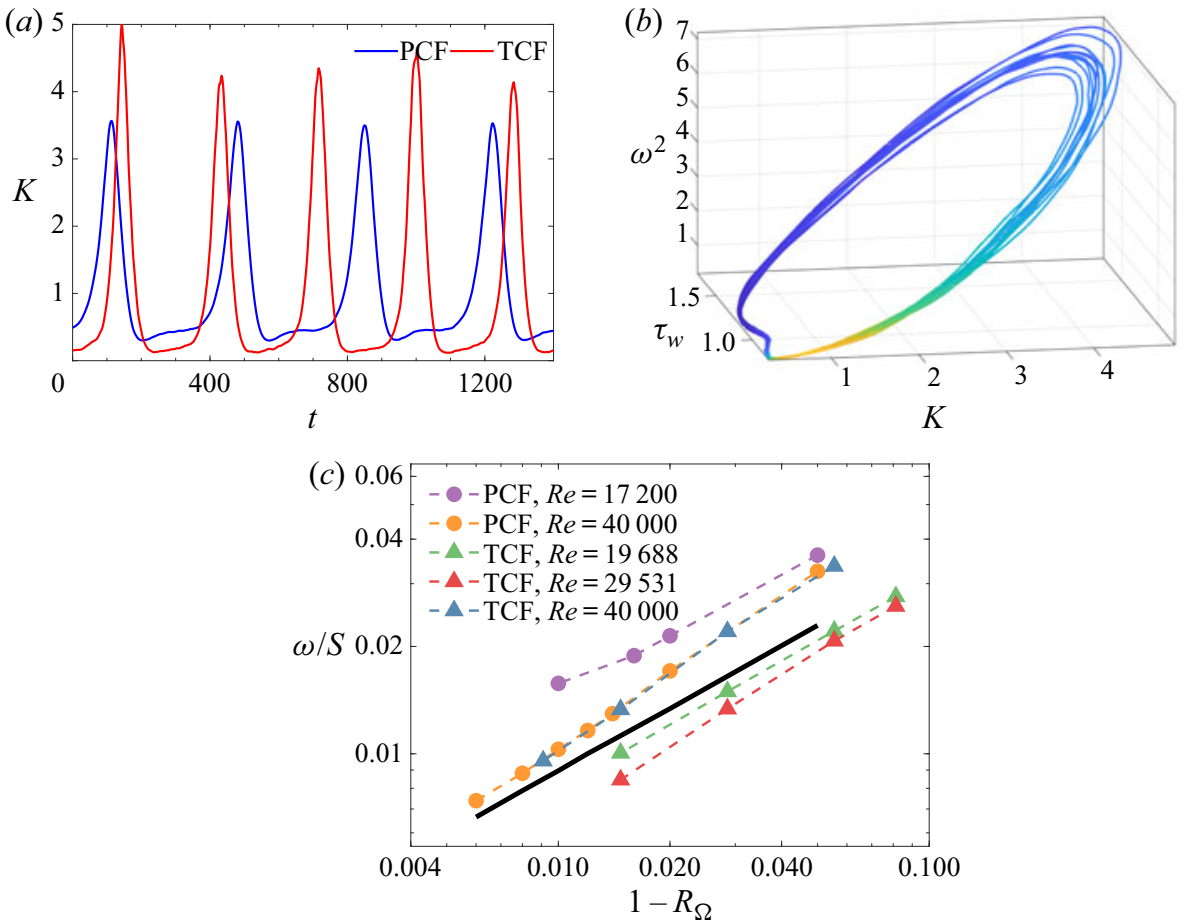

Figure 7. (a) Time series of $K$ in PCF at $R_{\Omega}=0.97$ and TCF at $R_{\Omega}=0.98$ and $R e=40000$. Time is non-dimensionalized by the shear rate $S=\Delta U / d$. (b) Phase space plot of volume integrated $K$ and enstrophy $\omega^{2}$, and wall shear stress $\tau_{w}$ in PCF at $R e=40000$ and $R_{\Omega}=0.99$. Yellow and blue colours indicate large and small temperature fluctuations, respectively. (c) Frequency of the bursts non-dimensionalized by $S$ for PCF and TCF. The black solid line gives the growth rate of the most unstable mode in PCF predicted by linear theory.

Couette flows are fully turbulent at higher $R e$ and also when HTE is maximal, leading to plume-like thermal structures (figure $6 c, d$ ). Above $R e \approx 10^{4}$, high values of HTE are accompanied by strong recurring low-frequency bursts of turbulence in both PCF and TCF. Such turbulent bursts are evident in time series of the volume-integrated turbulent kinetic energy $K$ (figure $7 a$ ) and emerge if $R_{\Omega} \gtrsim 0.94$. These are persistent and approximately periodic and come along with bursts of enstrophy and temperature fluctuations and significant changes of shear stresses and heat fluxes at the wall. A phase-space plot illustrates the approximate limit cycle dynamics (figure $7 b$ ). The burst frequency declines for $R_{\Omega} \rightarrow 1$ because the flow becomes more stable and follows a similar scaling in all cases (figure $7 c$ ). During the approximate limit cycle oscillations, PCF and TCF are supercritical and continuously though weakly turbulent between the bursts. I have verified that PCF is also linearly unstable if the mean velocity profile from the DNSs is used in the stability analysis, instead of the laminar one. The growth rate of the most unstable mode follows a similar trend as the burst frequency (figure $7 c$ ), suggesting that the bursts are related to linear instabilities.

\section{Concluding remarks}

The key conclusion of my study is that even a simple Coriolis body force can strongly change heat/mass transfer rates and can make heat/mass transfer much faster than momentum transfer in shear flows, as indicated theoretically recently (Alben 2017; Motoki 


\section{G. Brethouwer}

et al. 2018). Optimization of heat/mass transfer by body forces is thus a promising avenue for further research. The mechanism of momentum transport reduction by the Coriolis force does not depend on $R e$, implying that the observed dissimilarity between momentum and heat/mass transfer, found in both plane Couette and Taylor-Couette flow, persists at higher $R e$. The highest dissimilarity happens in rotating Couette flows close to the inviscid neutral stability state when momentum transfer is more strongly reduced than heat/mass transfer. Also, other rotating shear flows tend to evolve towards the neutral stability state (Métais et al. 1995; Barri \& Andersson 2010), suggesting that heat and mass are transported much faster than momentum in such flows. Dissimilarity between momentum and heat transport is also found in rotating channel flow (Matsubara \& Alfredsson 1996; Brethouwer 2018, 2019), albeit in a limited region where the flow approaches the zero-absolute-mean-vorticity state, and in shear flows with buoyancy forces (Li \& Bou-Zeid 2011; Pirozzoli et al. 2017). In DNS and rapid distortion theory of rotating uniformly sheared turbulence, Brethouwer (2005) observed turbulent Prandtl numbers much smaller than one when the zero-absolute-mean-vorticity state is approached, which also implies fast heat transport. This all suggests that other engineering and astrophysical flows also display dissimilarities between heat or mass transfer and momentum transfer. Another implication of the present study is that heat and mass transfer modelling in flows with body forces requires careful consideration since the Reynolds analogy can fail.

Supplementary material. Supplementary material is available at https://doi.org/10.1017/jfm.2020.1176.

Acknowledgements. The author is grateful to Professor G. Kawahara and Dr S. Motoki for the data on optimal transport in PCF, and to Bendiks Jan Boersma for providing and helping with the DNS code for TCF.

Funding. SNIC is acknowledged for providing computational resources in Sweden. The author further acknowledges financial support from the Swedish Research Council (grant number 621-2016-03533).

Declaration of interests. The author reports no conflict of interests.

Author ORCIDs.

(D) Geert Brethouwer https://orcid.org/0000-0002-9819-2906.

\section{REFERENCES}

Alben, S. 2017 Improved convection cooling in steady channel flows. Phys. Rev. Fluids 2, 104501.

BAlbus, S.A. \& HAWLEY, J.F. 1998 Instability, turbulence, and enhanced transport in accretion disks. Rev. Mod. Phys. 70, 1-53.

BARRI, M. \& ANDERSSON, H.I. 2010 Turbulent flow over a backward-facing step. Part 1. Effects of anti-cyclonic rotation. J. Fluid Mech. 665, 382-417.

Birnsteil, T., Dullemond, C.P. \& Brauer, F. 2010 Gas- and dust evolution in protoplanetary disks. Astron. Astrophys. 513, A79.

Boersma, B.J. 2011 A 6th-order staggered compact finite difference method for the incompressible Navier-Stokes and scalar equations. J. Comput. Phys. 230, 4940-4954.

BRAUCKMANN, H.J. \& ECKHARDT, B. 2013 Direct numerical simulations of local and global torque in Taylor-Couette flow up to $R e=30000$. J. Fluid Mech. 718, 398-427.

Brauckmann, H.J., Salewski, M. \& Eckhardt, B. 2016 Momentum transport in Taylor-Couette flow with vanishing curvature. J. Fluid Mech. 790, 419-452.

BRETHERTON, C.S. \& PARK, S. 2009 A new moist turbulence paramterization in the Community Atmosphere Model. J. Clim. 22, 3422-3448.

BRETHOUWER, G. 2005 The effect of rotation on rapidly sheared homogeneous turbulence and passive scalar transport. J. Fluid Mech. 542, 305-342.

BRETHOUWER, G. 2017 Statistics and structure of spanwise rotating turbulent channel flow at moderate Reynolds numbers. J. Fluid Mech. 828, 424-458.

Brethouwer, G. 2018 Passive scalar transport in rotating turbulent channel flow. J. Fluid Mech. 844, $297-322$. 


\section{Much faster heat/mass than momentum transport}

BRETHOUWER, G. 2019 Influence of spanwise rotation and scalar boundary conditions on passive scalar transport in turbulent channel flow. Phys. Rev. Fluids 4, 014602.

Chevalier, M., Schlatter, P., Lundbladh, A. \& Henningson, D.S. 2014 A pseudo-spectral solver for incompressible boundary layer flows. Tech. Rep. TRITA-MEK 2007:07, KTH Mechanics, Stockholm, Sweden.

Dubrulle, B., Dauchot, O., Daviaud, F., Longaretti, P.-Y., Richard, D. \& Zahn, J.-P. 2005 Stability and turbulent transport in Taylor-Couette flow with analysis of experimental data. Phys. Fluids 17, 095103.

Eckhardt, B., Doering, C.R. \& Whitehead, J.P. 2020 Exact relations between Rayleigh-Bénard and rotating plane Couette flow in two dimensions. J. Fluid Mech. 903, R4.

Esser, A. \& Grossmann, S. 1996 Analytic expression for Taylor-Couette stability boundary. Phys. Fluids 8, 1814-1819.

Ezeta, R., Sacco, F., Bakhuis, D., Huisman, S.G., Ostilla-Mónico, R., Verzicco, R., Sun, C. \& LoHSE, D. 2020 Double maxima of angular momentum transport in small gap $\eta=0.91$ Taylor-Couette turbulence. J. Fluid Mech. 900, A23.

Fénot, M., Bertin, Y., Dorignac, E. \& LAlizel, G. 2011 A review of heat transfer between concentric rotating cylinders with or without axial flow. Intl J. Therm. Sci. 50, 1138-1155.

van Gils, D.P.M., Huisman, S.G., Bruggert, G.-W., Sun, C. \& Lohse, D. 2011 Torque scaling in turbulent Taylor-Couette flow with co- and counterrotating cylinders. Phys. Rev. Lett. 106, 024502.

Grossmann, S., Lohse, D. \& Sun, C. 2016 High-Reynolds number Taylor-Couette flow. Annu. Rev. Fluid Mech. 48, 53-80.

GuAn, X. \& GAmmie, C.F. 2009 The turbulent magnetic Prandtl number of MHD turbulence in disks. Astrophys. J. 697, 1901-1906.

Hassanzadeh, P., Chini, G.P. \& Doering, C.R. 2014 Wall to wall optimal transport. J. Fluid Mech. 751, 627-662.

Hesselgreaves, J.E. 2000 Rationalisation of second law analysis of heat transfer exchangers. Intl J. Heat Mass Transfer 43, 4189-4204.

Kaithakkal, A.J., Kametani, Y. \& Hasegawa, Y. 2020 Dissimilarity between turbulent heat and momentum transfer induced by a streamwise travelling wave of wall blowing and suction. J. Fluid Mech. 886, A29.

KawATA, T. \& Alfredsson, P.H. 2016 Turbulent rotating plane Couette flow: Reynolds and rotation number dependency of flow structure and momentum transport. Phys. Rev. Fluids 1, 034402.

KAYS, W.M. 1994 Turbulent Prandtl number - where are we? Trans. ASME: J. Heat Transfer 116, 284-295.

Kays, W.M., Crawford, M. \& Weigand, B. 2005 Convective Heat and Mass Transfer. McGraw-Hill.

KEIL, F.J. 2018 Process intensification. Rev. Chem. Engng 34, 135-200.

LAKE, A., REZAIE, B. \& BEYERLEIN, S. 2017 Review of district heating and cooling systems for a sustainable future. Renew. Sustain. Energy Rev. 67, 417-425.

LeE, M. \& Moser, R.D. 2015 Direct numerical simulation of turbulent channel flow up to $R e_{\tau} \approx 5200$. J. Fluid Mech. 774, 395-415.

Li, D. \& BoU-ZEID, E. 2011 Coherent structures and the dissimilarity of turbulent transport of momentum and scalars in the unstable atmospheric surface layer. Boundary-Layer Meteorol. 140, 243-262.

Matsubara, M. \& Alfredsson, P.H. 1996 Experimental study of heat and momentum transfer in rotating channel flow. Phys. Fluids 8, 2964-2973.

Métais, O., Flores, C., Yanase, S., Riley, J.J. \& Lesieur, M. 1995 Rotating free-shear flows. Part 2. Numerical simulations. J. Fluid Mech. 293, 47-80.

Motoki, S., Kawahara, G. \& Shimizu, M. 2018 Optimal heat transfer enhancement in plane Couette flow. J. Fluid Mech. 835, 1157-1198.

NagATA, M. 1990 Three-dimensional finite-amplitude solutions in plane Couette flow: bifurcation from infinity. J. Fluid Mech. 217, 519-527.

Nemri, M., Charton, S. \& Climent, E. 2016 Mixing and axial dispersion in Taylor-Couette flows: the effect of the flow regime. Chem. Engng Sci. 139, 109-124.

Ostilla, R., Stevens, R.J.A.M., Grossmann, S., Verzicco, R. \& Lohse, D. 2013 Optimal Taylor-Couette flow: direct numerical simulations. J. Fluid Mech. 719, 14-46.

Ostilla-Mónoci, R., VAn der Poel, E.P., Verzicco, R., Grossmann, S. \& Lohse, D. $2014 a$ Exploring the phase diagram of fully turbulent Taylor-Couette flow. J. Fluid Mech. 761, 1-26.

Ostilla-Mónoci, R., Verzicco, R., Grossmann, S. \& Lohse, D. 2014b Turbulence decay towards the linearly stable regime of Taylor-Couette flow. J. Fluid Mech. 748, R3. 


\section{G. Brethouwer}

Peeters, J.W.R., Pecnik, R., Rohde, M., VAn der Hagen, T.H.J.J. \& Boersma, B.J. 2016 Turbulence attenuation in simultaneously heated and cooled annular flows at supercritical pressures. J. Fluid Mech. 799, 505-540.

Pirozzoli, S., Bernardini, M., Verzicco, R. \& Orlandi, P. 2017 Mixed convection in turbulent channels with unstable stratification. J. Fluid Mech. 821, 482-516.

Pirozzoli, S., Bernardini, M. \& ORlandi, P. 2016 Passive scalars in turbulent channel flow at high Reynolds number. J. Fluid Mech. 788, 614-639.

Reynolds, O. 1874 On the extent and action of the heating surface of steam boilers. Proc. Lit. Phil. Soc. Manchester 14, 7-12.

Salewski, M. \& ECKhardT, B. 2015 Turbulent states in plane Couette flow with rotation. Phys. Fluids 27, 045109.

Souza, A.N., Tobasco, I. \& Doering, C.R. 2020 Wall-to-wall optimal transport in two dimensions. J. Fluid Mech. 889, A34.

WEBB, R.L. 1981 Performance evaluation criteria for use of enhanced heat transfer surfaces in heat exchanger design. Intl J. Heat Mass Transfer 24, 715-726.

YAmamoto, A., Hasegawa, Y. \& KASAgi, N. 2013 Optimal control of dissimilar heat and momentum transfer in a fully developed turbulent channel flow. J. Fluid Mech. 733, 189-220.

Zhang, S., XiA, Z., Shi, Y. \& CHEn, S. 2019 A two-dimensional-three-component model for spanwise rotating plane poiseuille flow. J. Fluid Mech. 880, 478-496.

Zhu, X., Stevens, R.J.A.M., Shishrina, O., Verzicco, R. \& Lohse, D. $2019 N u \sim R a^{1 / 2}$ scalings enabled by multiscale wall roughness in Rayleigh-Bénard turbulence. J. Fluid Mech. 869, R4.

Ziefuss, M. \& MEHDiZADEH, A. 2020 A comprehensive assessment of the Reynolds analogy in predicting heat transfer in turbulent wall-bounded shear flows. Intl J. Heat Fluid Flow 81, 108527. 